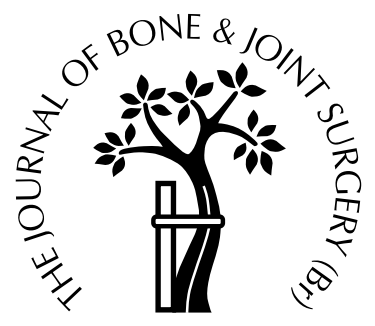

\title{
The relationship between the strength of supination of the forearm and rotation of the shoulder
}

\author{
N. Savva, C. J. P. McAllen, G. E. B. Giddins \\ From the Royal United Hospital and the University of Bath, England
}

$\mathbf{I}^{\mathrm{n}}$ children with obstetric brachial plexus palsy (OBPP) who develop an internal rotation deformity of the shoulder, release of subscapularis improves the range of external rotation of the shoulder and the strength of supination of the forearm. We studied the strength of supination in 35 healthy adult volunteers at $45^{\circ}$ of both internal and external rotation.

The mean and maximum torques were greater in external than internal rotation by $8.7 \%$ and $7.5 \%$, respectively. This was highly significant $(p<0.0001)$.

The increased strength of supination in external rotation is probably because the maximum power of biceps, particularly the long head, may be exerted in this position. In children the difference may be even greater due to anatomical differences causing the dramatic increases in the strength of supination after surgery for OBPP.

In adults our findings suggest that the supination exercises which are undertaken after injury or surgery to the forearm or wrist should be performed in external rotation.

J Bone Joint Surg [Br] 2003;85-B:406-7.

Received 4 July 2002; Accepted 19 September 2002

Children who have an obstetric brachial plexus palsy of the upper trunk from birth may develop a fixed internal rotation deformity of the glenohumeral joint. Release of the subscapularis can be performed to increase external rotation. ${ }^{1}$ This study was prompted by the observation that these children also gain supination of the forearm after operation, and a similar effect has also been noted after rotational osteotomy of the proximal humerus. ${ }^{2}$

N. Savva, FRCS, Specialist Registrar in Trauma and Orthopaedics

C. J. P. McAllen, FRCS, Specialist Registrar in Trauma and Orthopaedics

G. E. B. Giddins, FRCS, EDHS, Consultant in Orthopaedics and Hand Surgery

Department of Orthopaedics and Trauma, Royal United Hospital, Combe Park, Bath BA1 3NG, UK.

Correspondence should be sent to Mr G. E. B. Giddins.

(C)2003 British Editorial Society of Bone and Joint Surgery doi.10.1302/0301-620X.85B3.13651\$2.00
Materials and Methods

We recruited 35 volunteers, 19 men and 16 women, with a mean age of 39.9 years (24 to 61). Five reported pain, stiffness or weakness in one arm and these limbs were excluded from the study. None was aware of the hypothesis being tested. The concentric isokinetic strength of supination was assessed using the Kin-Com 125E Plus dynamometer (Chattanooga, Tennessee) allowing $60^{\circ}$ of rotation per second through $140^{\circ}$. The subjects could monitor their performance on a screen in order to maximise motor recruitment. The test was performed with the subject standing with the elbow flexed at $90^{\circ}$ and the humerus strapped to the side of the body in the anatomical position. Each subject was tested with the glenohumeral joint at $45^{\circ}$ of internal and external rotation by standing on a template with these angles marked and each was given two practices on the machine for each limb included. This was found to produce consistent results in a preliminary study. Three observations were performed in both internal and external rotation for each arm, in a random order to minimise the effect of fatigue. The mean and maximum torques throughout supination were recorded for each arm in each position. The mean of the results was expressed as a percentage difference between the strengths of supination and pronation.

The results were analysed using the two-tailed Student $t$ test.

Results

The mean and maximum torques of supination were greater in external than internal rotation by $8.7 \%(-25.5$ to +37.5$)$ and $7.5 \%(-27.7$ to +50.6$)$, respectively (Figs 1 and 2$)$. This was highly significant $(\mathrm{p} \leq 0.0001)$.

\section{Discussion}

The strength of supination is significantly greater at $45^{\circ}$ of external rotation than at $45^{\circ}$ of internal rotation of the glenohumeral joint.

Supination of the forearm is performed primarily by the supinator and biceps brachii muscles. Movement of the glenohumeral joint has no effect on the biomechanics of the supinator, but moving from internal to external rotation 


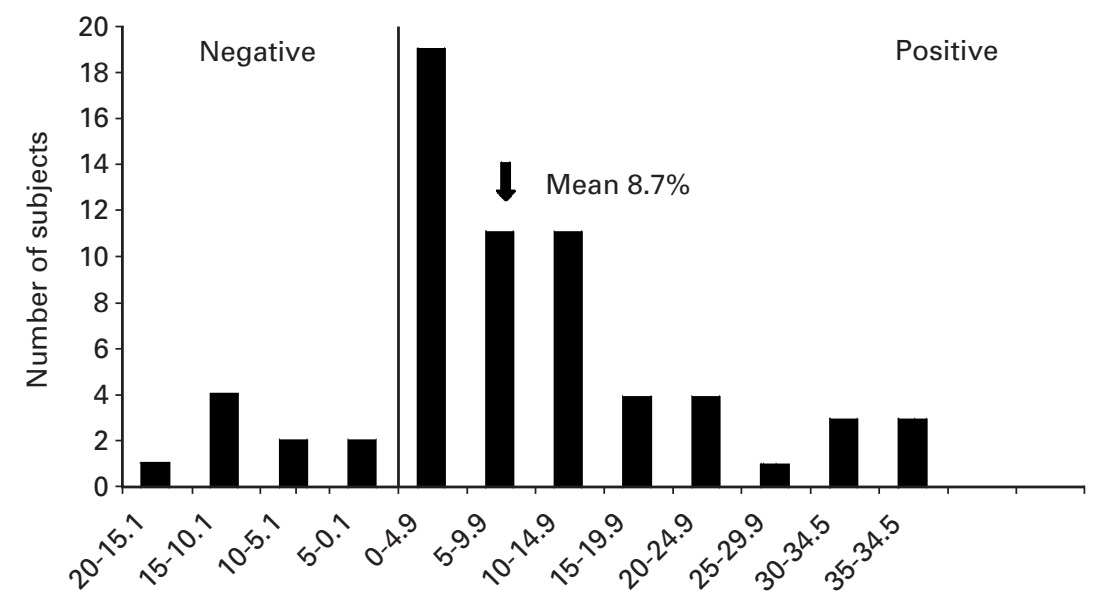

Fig. 1

The mean torque generated in external rotation minus that in internal rotation of the glenohumeral joint expressed as a percentage. To the right of the zero line are subjects in whom the power of supination was greater in external rotation of the glenohumeral joint than in internal rotation and to the left are those in whom it was vice versa.

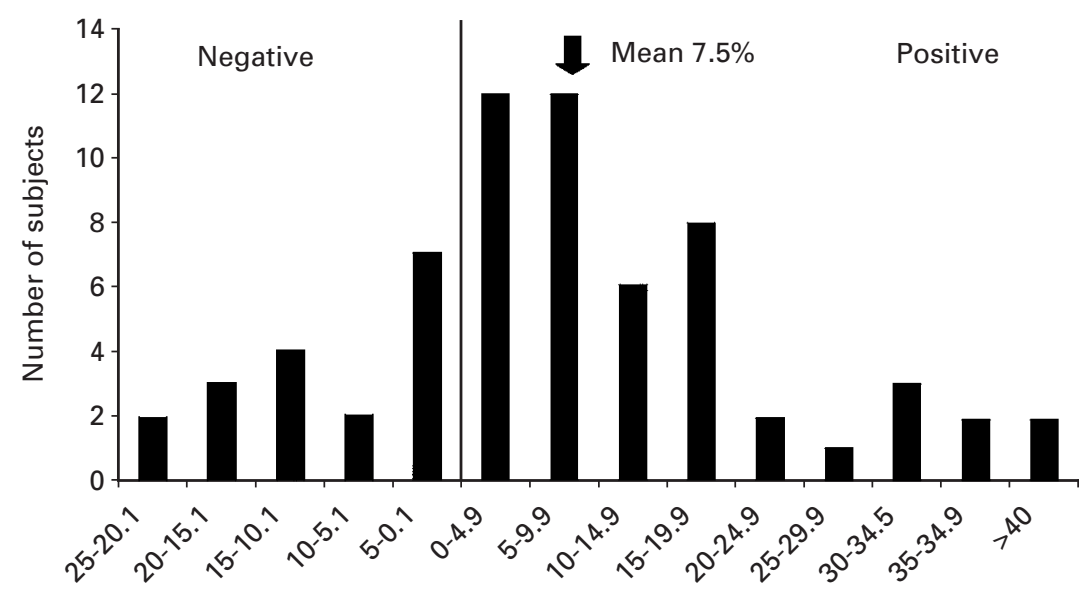

Fig. 2

The maximum torque generated in external rotation minus that in internal rotation of the glenohumeral joint expressed as a percentage. To the right of the zero line are subjects in whom the power of supination was greater in external rotation of the glenohumeral joint than in internal rotation and to the left are those in whom it was vice versa.

draws the long head of biceps over the head of the humerus lengthening the muscle belly thus maximising its power: length ratio. ${ }^{3}$

This explains the observation that release of subscapularis may increase the power of supination in children with obstetric brachial plexus palsy of the upper trunk further validating it as a therapeutic procedure. It also has implications for rehabilitation of the elbow and wrist after injury or surgery. Exercises which are aimed at improving the strength and range of movement of supination can be more effectively performed with the glenohumeral joint in external rotation.
We wish to thank G. Hunter of the Human Analysis Laboratory, Glenside, Bristol and G. Taylor, Statistician at the Royal United Hospital, Bath for their help.

No benefits in any form have been received or will be received from a commercial party related directly or indirectly to the subject of this article.

\section{References}

1. Sever JW. The results of a new operation for obstetric paralysis. Am J Orthop Surg 1918;16:248.

2. Kirkos JM, Papadopoulos IA. Late treatment of brachial plexus palsy secondary to birth injuries: rotational osteotomy of the proximal part of the humerus. J Bone Joint Surg [Am] 1998;80-A:1477-83.

3. Winters JM, Klevveno DG. Effect of initial upper-limb alignment on muscle contributions to isometric strength curves. J Biomech 1993;26:143-53 